\title{
The role of miR156 in developmental transitions in Nicotiana tabacum
}

\author{
ZHANG TianQi ${ }^{1,2}$, WANG JiaWei ${ }^{1} \&$ ZHOU ChuanMiao $^{1 *}$ \\ ${ }^{1}$ National Key Laboratory of Plant Molecular Genetics $(N K L P M G)$, Institute of Plant Physiology and Ecology, Shanghai Institutes for \\ Biological Sciences (SIBS), Shanghai 200032, China; \\ ${ }^{2}$ University of Chinese Academy of Sciences, Shanghai 200032, China
}

Received November 10, 2014; accepted December 16, 2014; published online February 11, 2015

\begin{abstract}
Plants undergo a series of developmental transitions during their life cycle. After seed germination, plants pass through two distinct phases: the vegetative phase in which leaves are produced and the reproductive phase in which flowering occurs. Based on the reproductive competence and morphological changes, the vegetative phase can be further divided into juvenile and adult phases. Here, we demonstrate that the difference between juvenile and adult phase of Nicotiana tabacum is characterized by the changes in leaf size, leaf shape as well as the number of leaf epidermal hairs (trichomes). We further show that miR156, an age-regulated microRNA, regulates juvenile-to-adult phase transition in N. tabacum. Overexpression of miR156 results in delayed juvenile-to-adult transition and flowering. Together, our results support an evolutionarily conserved role of miR156 in plant developmental transitions.
\end{abstract}

phase transition, miR156, Nicotiana tabacum

Citation: Zhang TQ, Wang JW, Zhou CM. The role of miR156 in developmental transitions in Nicotiana tabacum. Sci China Life Sci, 2015, 58: 253-260, doi: $10.1007 / \mathrm{s} 11427-015-4808-5$

Plants undergo a series of developmental transitions during their life cycle. The first transition is seed germination, in which the embryonic development is switched to the postembryonic mode of growth. One hallmark of germination is the activation of shoot apical meristem (SAM), a population of pluripotent stem cells responsible for producing all the above-ground lateral organs including stems, leaves and flowers. The seedling then passes through a vegetative phase, where SAM gives rise to leaves, followed by the transition to the reproductive phase, where SAM produces flowers [1].

Vegetative phase change refers to the transition from juvenile to adult phase. This transition is marked by changes in morphology and an increase in reproductive competence. Unlike plants in juvenile phase, the plants in adult phase are

*Corresponding author (email: yczhang@ sibs.ac.cn) able to flower in response to endogenous signals such as sugar and hormone, as well as environmental factors such as available nutrients, day length, light intensity, light quality, and ambient temperature [2-5]. Although the morphological features that distinguish these two phases differ between species, they usually include leaf shape and size, leaf arrangement, epidermal cell differentiation pattern, the regenerative capacity for adventitious root and the length of internode [6-8].

MicroRNAs (miRNAs) are a class of 20-24 nucleotide endogenous RNAs that usually regulate targeted gene expression by transcript cleavage or translational inhibition [9]. miRNAs are widely distributed in animals and plants [10]. Recently, many miRNAs have been identified in a unicellular green alga, Chlamydomonas reinhardtii and a multicellular species, Volvox carteri, suggesting that miRNAs play regulatory roles in the biology of green algae [11-13]. Re- 
cent studies have revealed that miR156 plays critical roles in the developmental transitions including vegetative phase transition and flowering [14-17]. Consistently being one of the evolutionarily conserved miRNAs in plants from moss to higher plants, miR156 regulates the juvenile-to-adult phase transition in many plant species, including Arabidopsis thaliana, Arabis alpina, Brassica rapa, Cardamine flexuosa, Populus x canadensis, Solanum tuberosum (potato), Solanum lycopersicum (tomato), and Zea mays [18-24]. Overexpression of miR156 prolongs juvenile phase, whereas inactivation of miR156 results in the opposite phenotype. The role of miR156 in vegetative phase transition is correlated to its temporal expression pattern. It has been shown that the mature miR156 is highly enriched in seedlings and gradually decreased with time [25-29]. miR156 overexpression also causes a severe delay in flowering, especially under non-inductive condition [23,25,30].

The targets of miR156 belong to a group of plant-specific transcription factors, called SQUAMOSA PROMOTER BINDING PROTEIN (SBP)-box genes [31]. In A. thaliana, 11 out of 17 SBP-box genes are targeted by miR156 [32]. These miR156-targeted SPLs can be structurally divided into two groups, represented by SPL3 and SPL9 [32]. SPL9 protein is larger than SPL3 because it harbors a carboxyl-terminal domain with unknown function. The biological role of SPL3 and SPL9 group genes in A. thaliana is divergent. For example, SPL9 and SPL15 redundantly regulate leaf initiation rate, leaf size and shoot maturation [19,25,33-35], whereas SPL3 temporally regulates floral transition $[26,26,36]$.

In this manuscript, we presented, for the first time to our knowledge, the characterization of developmental transitions in Nicotiana tabacum. Moreover, we found that the level of miR156 determines the timing of juvenile-to-adult phase transition and flowering in $N$. tabacum.

\section{Materials and methods}

\subsection{Plant materials}

N. tabacum L. cv. SR1 was grown at $21^{\circ} \mathrm{C}$ (day) $/ 19^{\circ} \mathrm{C}$ (night) in long day (16 h light $/ 8 \mathrm{~h}$ dark). 35S::MIM156 and $35 S:: M I R 156$ constructs were described [25]. For transgenic $N$. tabacum plants, the overnight culture of A. tumefaciens was resuspended with infection buffer $\left(30 \mathrm{~g} \mathrm{~L}^{-1}\right.$ glucose) to $A_{600}=0.8$. Tobacco seeds were sterilized with $20 \% \mathrm{NaClO}$ for $15 \mathrm{~min}$ and geminated on MS plate (4.4 g MS basal medium w/vitamins powder (PhytoTechnonolgy Laboratories, USA), $0.5 \mathrm{~g} \mathrm{~L}^{-1}$ methylester sulfonate, $20 \mathrm{~g} \mathrm{~L}^{-1}$ sucrose, $8 \mathrm{~g}$ $\mathrm{L}^{-1}$ agar, $\mathrm{pH}$ 7.5). Leaf dics ( $1 \mathrm{~cm}$ in diameter) was infected with A. tumefaciens suspension for $30 \mathrm{~min}$. The explants were briefly dried and transferred to sterile filter papers and kept in dark at $25^{\circ} \mathrm{C}$ for $2 \mathrm{~d}$. The explants were then transferred to selection shooting-medium (4.4 g MS basal me- dium w/vitamins powder, $0.5 \mathrm{~g} \mathrm{~L}^{-1}$ methylester sulfonate, $20 \mathrm{~g} \mathrm{~L}^{-1}$ sucrose, $2 \mu \mathrm{g} \mathrm{mL}^{-1}$ 6-BA, $100 \mu \mathrm{gL}^{-1}$ kanamycin and $250 \mu \mathrm{g} \mathrm{mL}^{-1}$ timentin, $8 \mathrm{~g} \mathrm{~L}^{-1}$ agar, $\mathrm{pH}$ 5.7) and incubate at $25^{\circ} \mathrm{C}$ for 2 weeks under long day condition. The explants were subcultured at a 3-week interval. The whole explants together with the shoots were transferred to selection rooting-medium (4.4 g MS basal medium w/vitamins powder, $0.5 \mathrm{~g} \mathrm{~L}^{-1}$ methylester sulfonate, $20 \mathrm{~g} \mathrm{~L}^{-1}$ sucrose, $100 \mu \mathrm{g} \mathrm{mL}{ }^{-1}$ kanamycin and $250 \mu \mathrm{g} \mathrm{mL}^{-1}$ timentin, $8 \mathrm{~g} \mathrm{~L}^{-1}$ agar, pH 5.7). Rooted plants were transferred to soil and grown in growth chamber. Independent T2 lines were used for regeneration assays.

\subsection{Cloning of NtSPLs in N. tabacum}

The coding sequences of NtSPLs were obtained by RACE method. Briefly, total RNAs of flowers were reverse- transcribed using a GeneRacer ${ }^{\mathrm{TM}}$ Oligo dT Primer (Invitrogen, 5'-GCTGTCAACGATACGCTACGTAACGGCATGACA-

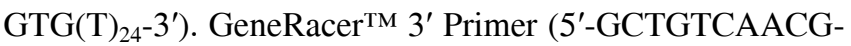
ATACGCTACGTAACG-3') and GeneRac- er ${ }^{\mathrm{TM}} 3^{\prime}$ Nested Primer (5'-CGCTACGTAACGGCATGACAGTG-3') served as 3' RACE primer. To design 5' RACE primers, protein sequences of AtSPLs were used to BLAST in N. tabacum EST database in NCBI (http://blast.ncbi.nlm. nih.gov/ Blast.cgi). The most similar nucleotide sequences were used to design gene-specific 5' RACE primer. The primary PCR conditions were $94^{\circ} \mathrm{C}$ for $30 \mathrm{~s}, 72^{\circ} \mathrm{C}$ for 5 min (5 cycles); $94^{\circ} \mathrm{C}$ for $30 \mathrm{~s}, 70^{\circ} \mathrm{C}$ for $5 \mathrm{~min}\left(5\right.$ cycles); $94^{\circ} \mathrm{C}$ for $15 \mathrm{~s}$, $64^{\circ} \mathrm{C}$ for $15 \mathrm{~s}, 72^{\circ} \mathrm{C}$ for $5 \mathrm{~min}$ ( 22 cycles), while the secondary PCR conditions were $94^{\circ} \mathrm{C}$ for $15 \mathrm{~s}, 58^{\circ} \mathrm{C}$ for $30 \mathrm{~s}$, and $72^{\circ} \mathrm{C}$ for $5 \mathrm{~min}$ (30 cycles). The amplified fragments were ligated into pMD18-T vector (TaKaRa, Japan) and sequenced.

\subsection{Phenotypic analyses}

For leaf size and shape measurement, the fully expanded leaves of wild type, 35S::MIR156 and 35S::MIM156 plants were detached. The length and width of blade were measured.

Chlorophyll content measurement was performed according to the method described previously [37]. Briefly, $200 \mathrm{mg}$ fresh leaves were grounded and chlorophyll was extracted with $2 \mathrm{~mL} 80 \%$ acetone. The chlorophyll content was calculated using spectrophotometric absorbance $(A)$ at light wavelength of 663 and $645 \mathrm{~nm}$. Chlorophyll a $(\mathrm{mg}$ $\left.\mathrm{g}^{-1}\right)=12.7 \times A_{663}-2.69 \times A_{645} ;$ Chlorophyll b $\left(\mathrm{mg} \mathrm{g}^{-1}\right)=22.9 \times$ $A_{645}-4.86 \times A_{663} ;$ Chlorophyll a+b $\left(\mathrm{mg} \mathrm{g}^{-1}\right)=8.02 \times A_{663}+$ $20.20 \times A_{645}$. Three independent experiments were performed.

For leaf trichome analyses, the fully expanded leaves of the plants of different ages were detached, frozen with liquid nitrogen, and examined by SEM. 
For flowering time measurement, the seeds were geminated on soil. Plants were then grown in long day. The numbers of leaves on the stem when the first floral buds were visible were scored.

\subsection{RNA gel blot}

The leaves from the plants of different ages were collected. To avoid the impact of leaf age on gene expression, the leaves of the same size ( $1 \mathrm{~cm}$ in length) were used for RNA analyses. Total RNA was extracted with Trizol reagent (Invitrogen, USA). Biological triplicates with technical duplicates were performed. Total RNA was separated on a $15 \%$ denaturing polyacrylamide/TBE/8 M urea gel and then transferred to Hybond-N+ membrane (GE Biosciences, USA). The complementary oligonucleotide of miR156 was end-labeled with $\left[\gamma_{-}{ }^{32} \mathrm{P}\right]$ ATP by T4 polynucleotide kinase (New England Biolabs, USA). Hybridization was performed overnight at $37^{\circ} \mathrm{C}$ in $5 \mathrm{~mL}$ of PerfectHyb hybridization buffer (Sigma, USA). Membranes were then washed three times with $2 \times$ SSC containing $0.1 \%$ SDS and exposed to Kodak MS film.

\subsection{Sequence alignment and phylogenetic analysis}

BLASTp was performed using the deduced amino acid sequence from NtSPLs and SlySBPs. Multiple sequence alignments and phylogenetic tree were generated by MEGA 5.0 [38]. Neighbor-joining (NJ) method was used to draw phylogenetic trees [39]. The accession numbers for NtSPLs were: NtSPL2, KP260634; NtSPL4, KP260635; NtSPL9, KP260636. The accession numbers for SlySBPs were: SlySBP10, Solyc05g015510; SlySBP12a, Solyc01g068100; SlySBP12b, Solyc05g053240; SlySBP2, Solyc04g045560; SlySBP6c, Solyc12g038520; SlySBP10, Solyc05g0155110; SlySBP13, Solyc05g015840; SlySBP6a, Solyc03g114850; SlySBP8a, Solyc10g018730; SlySBP8b, Solyc01g090730; SlySBP3, Solyc10g009080; SlySBP6b, Solyc05g012040; SlySBP15, Solyc10g078700; SlySBP4, Solyc07g053810; SlySBP7, Solyc01g080670.

\section{Results}

\subsection{The vegetative phase transition in N. tabacum}

To examine the morphological changes during vegetative phase in $N$. tabacum, we grew wild type plants under long day condition (16 h light $/ 8 \mathrm{~h}$ dark) at $21^{\circ} \mathrm{C}$ (day) $/ 19^{\circ} \mathrm{C}$ (night). We measured the following morphological features: leaf size, leaf shape and the number of trichomes. The fully expanded leaves from the plants of different ages were collected and the length and width of blades were measured (Figure 1A). The leaf size was progressively increased during development (Figure 1B). Compared to the 1st leaf, both blade length and width were doubled in the 2 nd leaf.
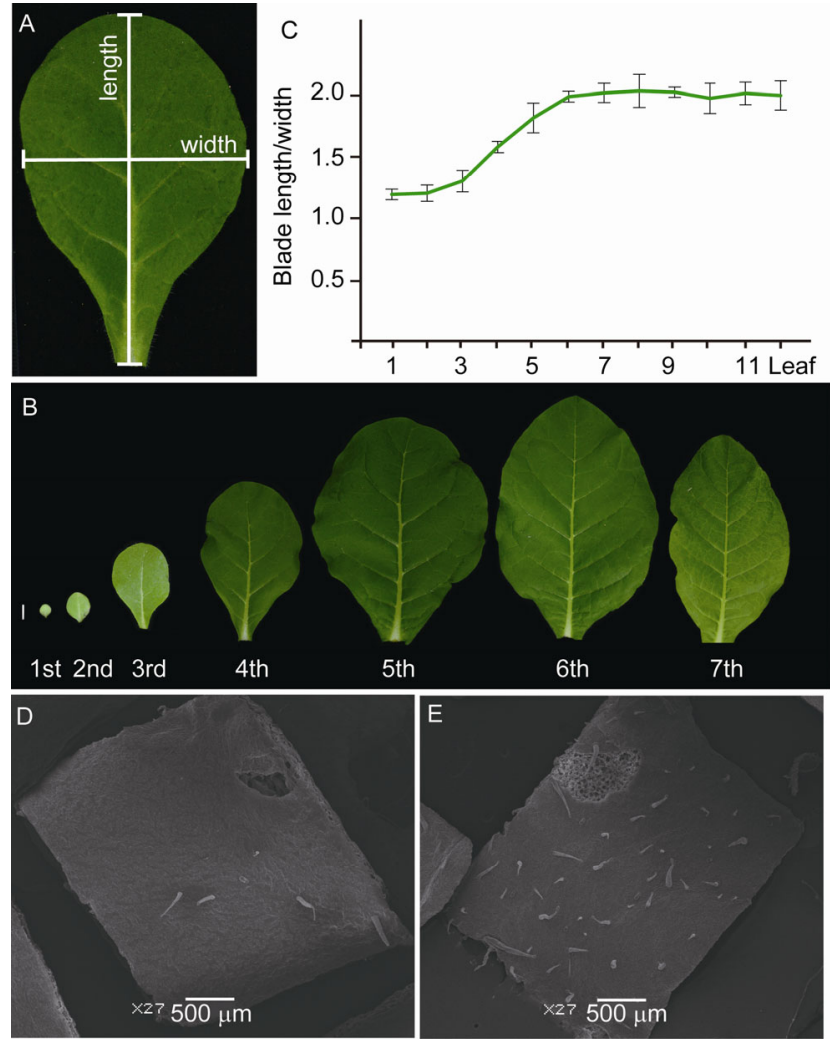

Figure 1 The developmental changes during vegetative phase. A, Schematic diagram of blade length/width ratio. B, Fully expanded leaves. The 1 st to the 7 th leaves were shown. C, Blade length/width ratio. $n=16$. Error bars stand for SD. D and E, SEM analyses of the adaxial leaf epidermis of the 1st leaf (D) and the 6th leaf (E). Bars stand for $500 \mu \mathrm{m} . n=8$.

The change in leaf size was accompanied by a change in leaf shape. The blade length/width ratio (leaf index) was 1.19 in early leaves (leaf \#1-2), and 2.00 in late leaves (leaf \#6-10) (Figure 1C).

Trichomes, as a model system for study of cell differentiation, have been reported to be associated with vegetativephase changes in A. thaliana: The leaf trichome distribution changes gradually from adaxial to abaxial as the shoot grows up [40,41]. While Arabidopsis trichomes are unicellular, the leaf of $N$. tabacum was covered with two types of multicellular trichomes-glandular and nonglandular. Compared to non-glandular trichome, the glandular trichome has bulge terminal at the tip which produces various alkaloids, terpenoids and defensive proteins $[42,43]$. To examine the distribution pattern of leaf trichomes at different phases in N. tabacum, we performed scanning electron microscope (SEM) analyses on the early and late leaves. We found that $85 \%$ of trichomes on $N$. tabacum leaf epidermis were glandular trichomes. Unlike Arabidopsis, both abaxial and adaxial sides of $N$. tabacum leaves produced trichomes. The number of trichomes was less in early leaf (leaf \#1-2) than in late leaf (leaf \#6-7) (Figure 1D and E). Taken together, these results indicated that the changes 
during vegetative phase in $N$. tabacum are characterized by the changes in leaf size, leaf shape, and the number of trichomes.

\section{2 miR156 regulates vegetative phase transition}

As mentioned earlier, miR156 acts as the master regulator of juvenility in many plant species. In N. tabacum, three expressed sequence tags (ESTs) encoding miR156-targeted SPLs (named as NtSPL2, NtSPL4 and NtSPL9) were identified. The full-length cDNAs of these genes were cloned by rapid amplification of cRNA ends (RACE). Sequence comparison and phylogenetic analysis revealed that NtSPL2, NtSPL4 and NtSPL9 showed high sequence similarities to Solanum lycopersicon (tomato) SlySBP4, SlySBP15 and SlySBP10, respectively [44] (Figure S1 in Supporting Information). The miR156 binding sites were located in the $3^{\prime}$ untranslated region (UTR) in NtSPL4, and in the coding region of NtSPL2 or NtSPL9 (Figure S2 in Supporting Information). We investigated the expression pattern of miR156 in the leaves of N. tabacum plants of different ages. To avoid the impact of leaf age on miR156 expression, the leaf of the same size ( $1 \mathrm{~cm}$ in length) was used. We found that miR156 was accumulated at high level at seedling stage, and subsequently decreased (Figure 2A). After the appearance of the 7th leaf, miR156 level reached a basal level and did not decline afterwards. Thus, these results revealed that the level of miR156, as seen in other plant species, is correlated with age in N. tabacum.

To determine whether miR156 plays a role in vegetative phase transition in N. tabacum, we generated the transgenic $N$. tabacum plants that overexpressed either miR156 (35S::MIR156) or a target mimicry of miR156 (35S:: MIM156), in which miR156 activity is suppressed [45]. In total, 50 lines for $35 S:: M I R 156$ and four lines for $35 S::$ MIM156 were obtained. RNA gel blot revealed that miR156 was highly expressed in $35 S:: M I R 156$ plants but reduced in

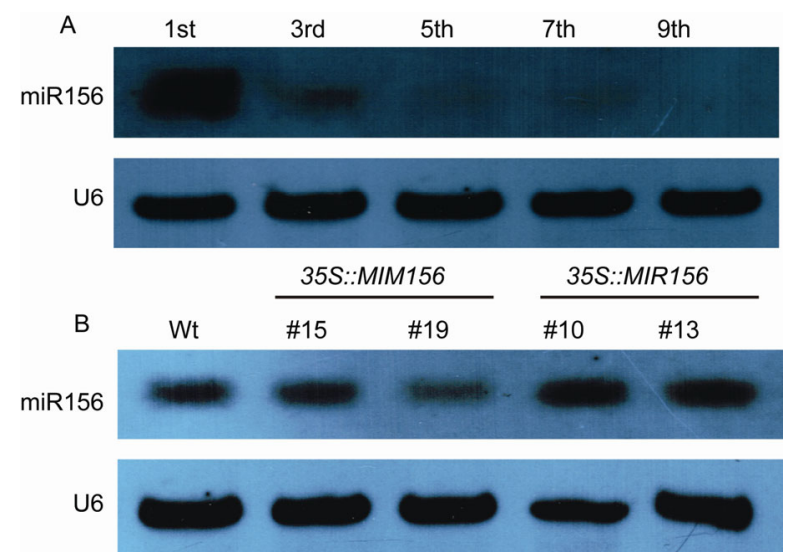

Figure 2 Molecular analyses of transgenic plants. A, RNA gel blot analysis of mature miR156 in wild type of different ages. U6 was blotted as loading control. B, Expression level of miR156 in wild type (Wt), $35 S: \because M I R 156$ and $35 S:: M I M 156$ transgenic plants. Two independent lines were examined.
35S::MIM156 (Figure 2B).

We compared the developmental changes during vegetative phase among wild type, 35S::MIR156 and $35 S::$ MIM156. The seeds were germinated at the same time and grew under the same condition. The level of miR156 was correlated with the leaf initiation rate. After germination, 35S::MIM156 slowly produced leaves, but the leaf initiation rate of $35 S:: M I R 156$ was increased (Figure 3A). In early leaves (the 1st to 3rd leaves), there was no difference in leaf size between wild type and $35 S:: M I R 156$ plants, whereas $35 S:: M I M 156$ had bigger leaves than wild type (Figure 3B and $\mathrm{C}$ ). The measurement of leaf index revealed that the shape of the 1st $35 S:: M I M 156$ leaf was oval, compared to the roundish leaf shape of wild type leaf at the same position (Figure 3D). In contrast, the leaf shape of 35S::MIR156 was nearly constant during development. SEM analyses indicated that $35 S:: M I M 156$ produces more trichomes than wild type in both early and late leaves, whereas $35 S:$ : MIR156 exerted an opposite effect (Figure 3E). Therefore, these results illustrated that overexpression of miR156 causes plants exhibiting juvenile characteristics, thereby delays juvenile-to-adult phase transition.

\section{3 miR156 negatively regulates flowering}

In A. thaliana, the role of miR156 in flowering is more pronounced in short days than in long days where the photoperiod plays a predominant role. Consistent with the fact that the $N$. tabacum plant (cv SR1) is a day neutral plant (the time of flowering is not affected by day length), overexpression of miR156 severely delayed flowering in long days. Fifteen of 50 T1 35S::MIR156 lines continued producing juvenile-type leaves and did not flower (Figure 4B). The rest $35 \mathrm{~T} 1$ plants eventually flowered. Compared to wild type, the flowering time was delayed by more than 20 leaves in $35 S:: M I R 156$ (Figure 4A and B). 35S::MIM156 caused early flowering phenotype, with 6 leaves less than wild type (Figure 4C). Thus, these observations indicated that miR156 plays a predominant role in flowering in $N$. tabacum, and is consistent with the notion that miR156 defines an endogenous flowering time pathway.

\section{4 miR156 participates in other developmental pro- cesses of $N$. tabacum}

The effect of miR156 on the development of $N$. tabacum is pleiotropic. miR156 overexpressor displayed the increased number of lateral shoots. Every node developed a lateral shoot in $35 S:: M I R 156$ plants, but not in wild type and $35 S:: M I M 156$ plants (Figure 4E). This phenotype is in agreement with previous findings in A. thaliana, rice and maize. The elevated level of miR156 leads to an increase in tillers or branches, whereas a point mutation in rice IPA1/ OsSPL14, which disturbs the miR156 recognition, results in a rice plant with a reduced tiller number [23,30,46-48]. 

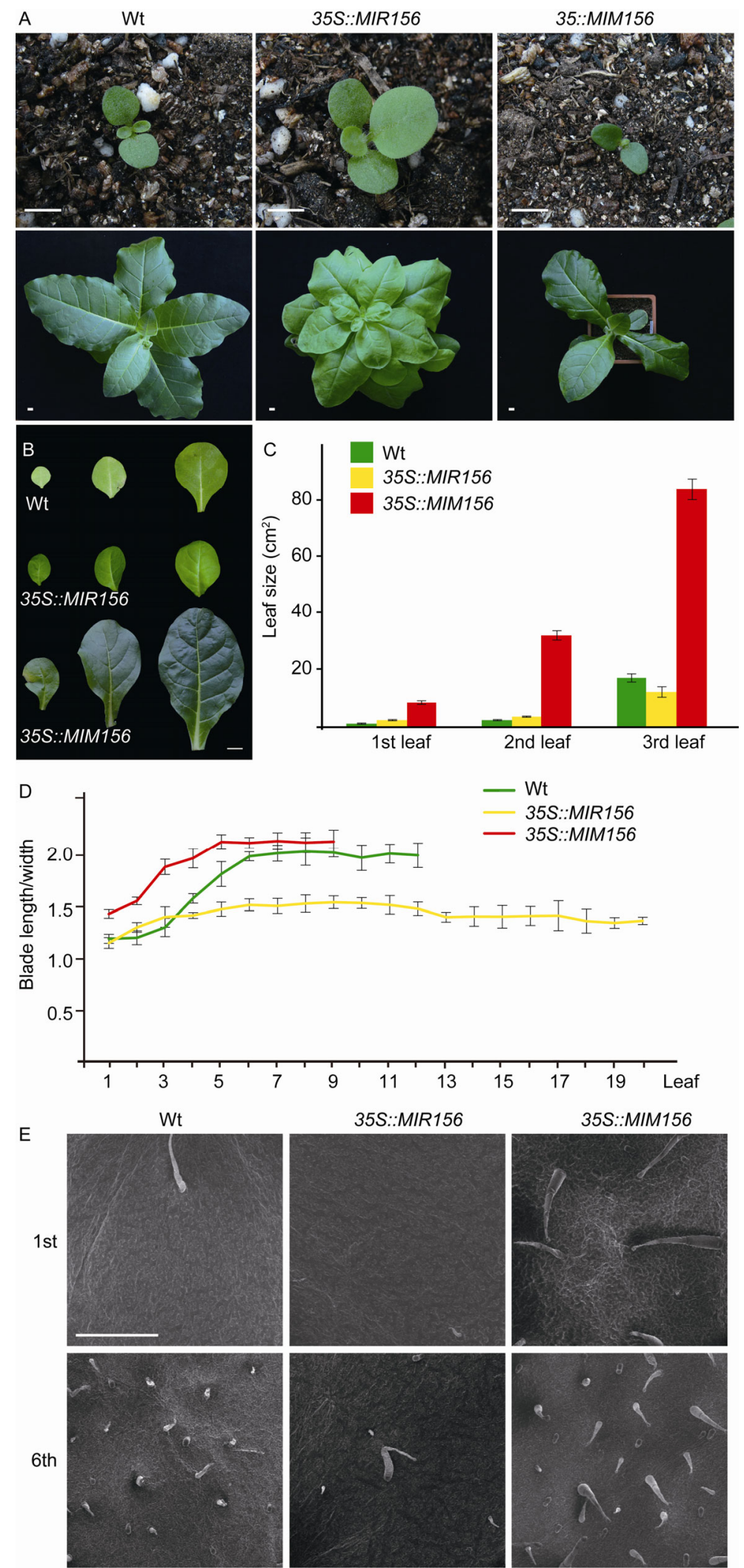

Figure 3 Phenotypic analyses of transgenic plants. A, Seedlings of wild type, 35S::MIR156 (\#13) and 35S::MIM156 (\#19) (top row);Adult plants of wild type, 35S::MIR156 (\#13) and 35S::MIM156 (\#19) (bottom row). Bars stand for $1.0 \mathrm{~cm}$. B, Fully expanded leaves of wild type, 35S::MIR156 (\#13) and $35 S:: M I M 156$ (\#19). Bars stand for $1.0 \mathrm{~cm}$. The 1 st to the $3 \mathrm{rd}$ leaves were shown. C, Leaf size of wild type, 35S::MIR156 (\#13) and 35S::MIM156 (\#19). $n=3$. Error bars stand for SD. D, Blade length/width ratio of wild type, 35S::MIR156 (\#13) and 35S::MIM156 (\#19). $n=5$. Error bars stand for SD. E, SEM analyses of abaxial leaf epidermis of wild type, 35S::MIR156 (\#13) and 35S::MIM156 (\#19). Bars stand for $500 \mu \mathrm{m} . n=8$. 


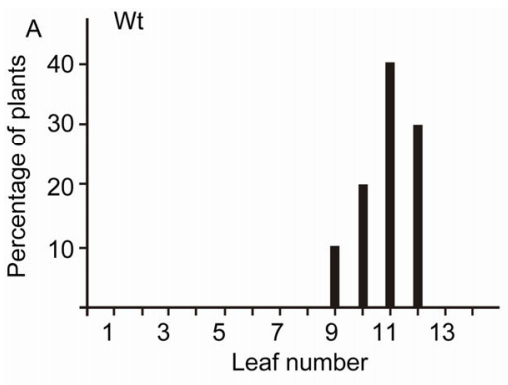

C

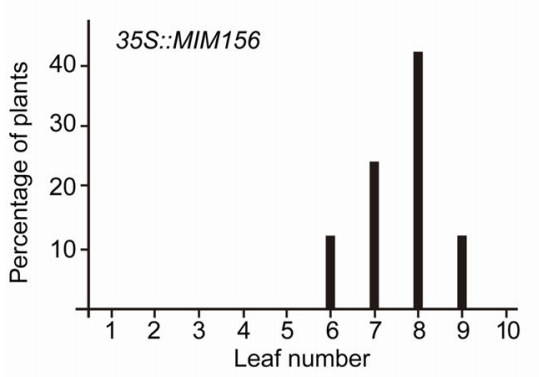

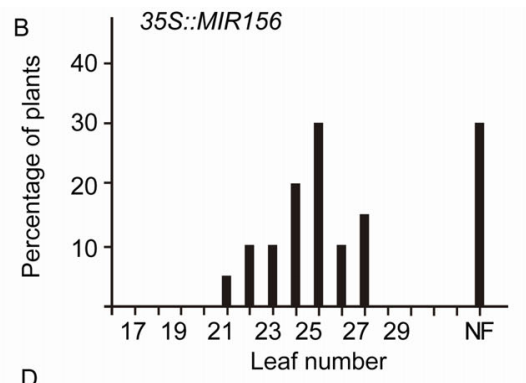

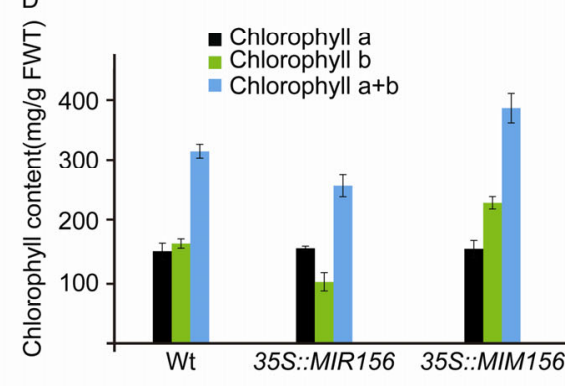

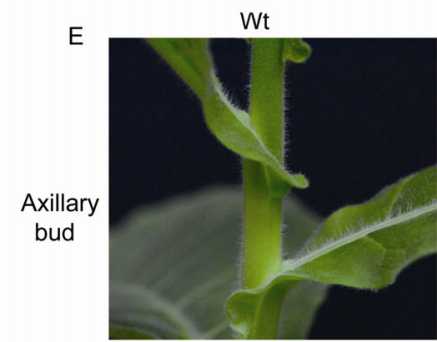

$\mathrm{F}$

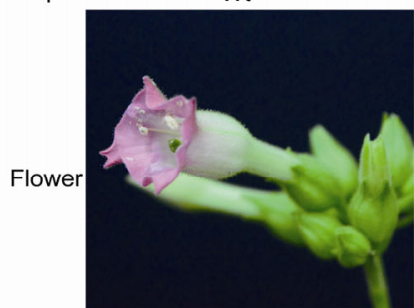

35S::MIR156

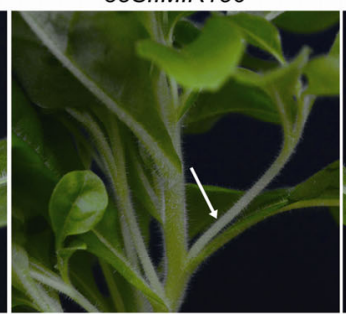

35S::MIR156

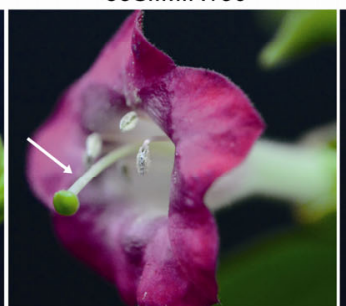

35S::MIM156

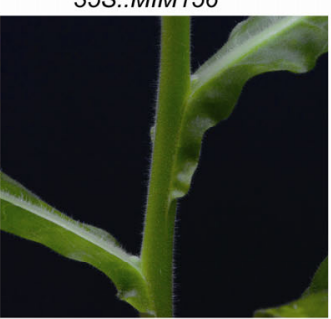

35S::MIM156

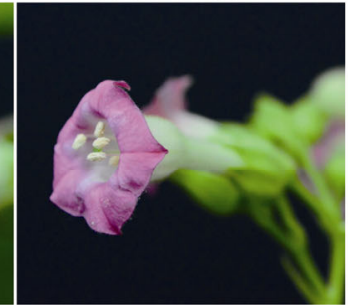

Figure 4 miR156 overexpression causes pleiotropic effects on development. A-C, Overexpression of miR156 delayed flowering in long days. The leaf numbers when plants started to flower were scored. Wild type (A) and a T2 line of 35S::MIM156 (\#19) (C) were examined. For 35S::MIR156, the flowering time of 50 independent T1 lines was measured. D, Measurements of chlorophyll a and b content in the leaves of wild type, 35S::MIR156 (\#13) and $35 S:: M I M 156$ (\#19). Error bars stand for SD. E, The increased number of lateral shoots in $35 S:: M I R 156$ (\#13) plants. Arrow indicates a developing lateral shoot in 35S::MIR156. F, Floral phenotype of wild type, 35S::MIR156 (\#13) and 35S::MIM156 (\#19) plants. Arrow indicates the decreased length of filaments in a $35 S:: M I R 156$ flower.

In A. thaliana, overexpression of miR156 did not cause obvious developmental defects in flowers. However, we did observe that miR156 overexpressor produced smaller flowers than wild type in $N$. tabacum. In addition, the length of filaments of flowers was decreased, which led to the reduced fertility (Figure 4F).

We also noticed that miR156 regulated leaf color. Compared to wild type, the leaf color of $35 S:: M I R 156$ plants was pale green, whereas that of $35 S: \because$ MIM156 plants was dark green. This difference is likely to be caused by the changes in chlorophyll content. Therefore, we measured the chlorophyll content of the 2nd leaves from wild type, $35 S:: M I R 156$ and $35 S:: M I M 156$ plants. Although there was no difference in chlorophyll a content, the level of chlorophyll $\mathrm{b}$ was increased in $35 S::$ MIM156 and reduced in 35S::MIR156 plants (Figure 4D).

\section{Discussion}

In A. thaliana, the formation of trichomes on the abaxial side of leaf is the one of the hallmarks for adult phase. However, we did not observe the same change in the trichome distribution pattern between early and late leaf in $N$. tabacum. Instead, we found that the number of trichomes differs between juvenile and adult phase. The late leaves have more trichomes than early leaves. Thus, these results 
suggest that the epidermal cell differentiation pattern may serve as a universal mark for juvenile-to-adult phase transition, although the morphological features of trichomes that distinguish these phases differs between species.

While A. thaliana miR156 overexpressor shows normal floral development [48], the elevated level of miR156 led to growth defects in flower development in N. tabacum (Figure $4 \mathrm{~F}$ ). Interestingly, a recent study revealed that overexpression of miR156 in tomato exhibited abnormal flower and fruit morphology, with fruits characterized by growth of extra carpels and ectopic structures [49], suggesting a Solanaceae-specific role of miR156-SPL in reproductive organ development.

Flowering time is controlled by both endogenous and exogenous cues such as day length and temperature. In $A$. thaliana, five genetic pathways have been identified, namely age, autonomous, gibberellin, photoperiod and vernalization pathway [50]. Age and photoperiod pathways induce floral transition through activation of flowering time integrators including $F T$, MADS-box genes and LEAFY ( $L F Y)$, whereas another three pathways promotes flowering through the removal of flowering repressors. In A. thaliana, photoperiod plays a predominant role in long days, whereas the function of miR156 in flowering is more evident under non-inductive short day conditions. Consistent with these observations, we found that miR156 acts as a major flowering time determinator in $N$. tabacum, a day neutral plant. Overexpression of miR156 results in a severe delay in flowering in long day condition. Thus, being the sole positive flowering promoter, the precise regulation of miR156 level is of great importance for the flowering time in day neutral plants.

We thank members in Wang JiaWei's lab for discussion and comments on the manuscript. We also thank Gao XiaoYan and Li JiQin for their assistance in SEM. This work was supported by grants from the National Natural Science Foundation of China (31400250), SIBS Young Investigator Program (2013KIP207) to Zhou ChuanMiao, and grants from the National Natural Science Foundation of China (31430013, 31222029, 912173023), State Key Basic Research Program of China (2013CB127000), Shanghai Pujiang Program (12PJ1409900), Recruitment Program of Global Experts (China), NKLPMG Key Research Program to Wang JiaWei.

1 Baurle I, Dean C. The timing of developmental transitions in plants. Cell, 2006, 125: 655-664

2 Srikanth A, Schmid M. Regulation of flowering time: all roads lead to Rome. Cell Mol Life Sci, 2011, 68: 2013-2037

3 Andres F, Coupland G. The genetic basis of flowering responses to seasonal cues. Nat Rev Genet, 2012, 13: 627-639

4 Amasino RM, Michaels SD. The timing of flowering. Plant Physiol, 2010, 154: 516-520

5 Kobayashi Y, Weigel D. Move on up, it's time for change-mobile signals controlling photoperiod-dependent flowering. Genes Dev, 2007, 21: 2371-2384

6 Poethig RS. Phase change and the regulation of developmental timing in plants. Science, 2003, 301: 334-336

7 Poethig RS. Vegetative phase change and shoot maturation in plants. Curr Top Dev Biol, 2013, 105: 125-152
8 Huijser P, Schmid M. The control of developmental phase transitions in plants. Development, 2011, 138: 4117-4129

9 Rogers K, Chen X. Biogenesis, turnover, and mode of action of plant microRNAs. Plant Cell, 2013, 25: 2383-2399

10 Bartel DP. MicroRNAs: target recognition and regulatory functions. 2009. Cell, 136: 215-233

11 Zhao T, Li G, Mi S, Li S, Hannon GJ, Wang XJ, Qi Y. A complex system of small RNAs in the unicellular green alga Chlamydomonas reinhardtii. Genes Dev, 2007, 21: 1190-1203

12 Molnar A, Schwach F, Studholme DJ, Thuenemann EC, Baulcombe DC. miRNAs control gene expression in the single-cell alga Chlamydomonas reinhardtii. Nature, 2007, 447: 1126-1129

13 Li J, Wu Y, Qi Y. MicroRNAs in a multicellular green alga Volvox carteri. Sci China Life Sci, 2014, 57: 36-45

14 Poethig RS. Small RNAs and developmental timing in plants. Curr Opin Genet Dev, 2009, 19: 374-378

15 Poethig RS. The past, present, and future of vegetative phase change. Plant Physiol, 2010, 154: 541-544

16 Chen X, Zhang Z, Liu D, Zhang K, Li A, Mao L. SQUAMOSA promoter-binding protein-like transcription factors: star players for plant growth and development. J Integr Plant Biol, 2010, 52: 946-951

17 Wang Y, Wu F, Bai J, He Y. BrpSPL9 (Brassica rapa ssp. pekinensis SPL9) controls the earliness of heading time in Chinese cabbage. Plant Biotechnol J, 2014, 12: 312-321

18 Wu G, Park MY, Conway SR, Wang JW, Weigel D, Poethig RS. The sequential action of miR156 and miR172 regulates developmental timing in Arabidopsis. Cell, 2009, 138: 750-759

19 Zhou CM, Zhang TQ, Wang X, Yu S, Lian H, Tang H, Feng ZY, Zozomova-Lihova J, Wang JW. Molecular basis of age-dependent vernalization in Cardamine flexuosa. Science, 2013, 340: 1097-1100

20 Wang JW, Park MY, Wang LJ, Koo Y, Chen XY, Weigel D, Poethig RS. miRNA control of vegetative phase change in trees. PLoS Genet, 2011, 7: e1002012

21 Bergonzi S, Albani MC, Ver Loren van Themaat E, Nordstrom KJ, Wang R, Schneeberger K, Moerland PD, Coupland G. Mechanisms of age-dependent response to winter temperature in perennial flowering of Arabis alpina. Science, 2013, 340: 1094-1097

22 Chuck G, Cigan AM, Saeteurn K, Hake S. The heterochronic maize mutant Corngrass1 results from overexpression of a tandem microRNA. Nat Genet, 2007, 39: 544-549

23 Eviatar-Ribak T, Shalit-Kaneh A, Chappell-Maor L, Amsellem Z, Eshed Y, Lifschitz E. A cytokinin-activating enzyme promotes tuber formation in tomato. Curr Biol, 2013, 23: 1057-1064

24 Wang JW, Czech B, Weigel D. miR156-regulated SPL transcription factors define an endogenous flowering pathway in Arabidopsis thaliana. Cell, 2009, 138: 738-749

$25 \mathrm{Wu}$ G, Poethig RS. Poethig, Temporal regulation of shoot development in Arabidopsis thaliana by miR156 and its target SPL3. Development, 2006, 133: 3539-3547

26 Yu S, Cao L, Zhou CM, Zhang TQ, Lian H, Sun Y, Wu JQ, Huang JR, Wang GD, Wang JW. Sugar is an endogenous cue for juvenile-to-adult phase transition in plants. eLIFE, 2013. 2: e00269

27 Yang L, Xu M, Koo Y, He J, Poethig RS. Sugar promotes vegetative phase change in Arabidopsis thaliana by repressing the expression of MIR156A and MIR156C. elife, 2013, 2: e00260

28 Xie K, Shen J, Hou X, Yao J, Li X, Xiao J, Xiong L. Gradual increase of miR156 regulates temporal expression changes of numerous genes during leaf development in rice. Plant Physiol, 2012, 158: $1382-1394$

29 Xie K, Wu C, Xiong L. Genomic organization, differential expression, and interaction of SQUAMOSA promoter-binding-like transcription factors and microRNA156 in rice. Plant Physiol, 2006, 142: 280-293

30 Cardon G, Hohmann S, Klein J, Nettesheim K, Saedler H, Huijser P. Molecular characterisation of the Arabidopsis SBP-box genes. Gene, 1999, 237: 91-104

31 Xing S, Salinas M, Hohmann S, Berndtgen R, Huijser P. miR156-targeted and nontargeted SBP-box transcription factors act 
in concert to secure male fertility in Arabidopsis. Plant Cell, 2010, 22: 3935-3950

32 Usami T, Horiguchi G, Yano S, Tsukaya H. The more and smaller cells mutants of Arabidopsis thaliana identify novel roles for SQUAMOSA PROMOTER BINDING PROTEIN-LIKE genes in the control of heteroblasty. Development, 2009, 136: 955-964

33 Schwarz S, Grande AV, Bujdoso N, Saedler H, Huijser P. The microRNA regulated SBP-box genes SPL9 and SPL15 control shoot maturation in Arabidopsis. Plant Mol Biol, 2008, 67: 183-195

34 Rubio-Somoza I, Zhou CM, Confraria A, Martinho C, von Born P, Baena-Gonzalez E, Wang JW, Weigel D. Temporal control of leaf complexity by miRNA-regulated licensing of protein complexes. Curr Biol, 2014, 24: 2714-2719

35 Jung JH, Ju Y, Seo PJ, Lee JH, Park CM. The SOC1-SPL module integrates photoperiod and gibberellic acid signals to control flowering time in Arabidopsis. Plant J, 2011, 69: 577-588

36 Ni Z, Kim ED, Ha M, Lackey E, Liu J, Zhang Y, Sun Q, Chen ZJ. Altered circadian rhythms regulate growth vigour in hybrids and allopolyploids. Nature, 2009, 457: 327-331

37 Thompson JD, Gibson TJ, Plewniak F, Jeanmougin F, Higgins DG. The CLUSTAL_X windows interface: flexible strategies for multiple sequence alignment aided by quality analysis tools. Nucleic Acids Res, 1997, 25: 4876-4882

38 Huson DH. SplitsTree: analyzing and visualizing evolutionary data. Bioinformatics, 1998, 14: 68-73

39 Balkunde R, Pesch M, Hulskamp M. Trichome patterning in Arabidopsis thaliana from genetic to molecular models. Curr Top Dev Biol, 2010, 91: 299-321

40 Telfer A, Bollman KM, Poethig RS. Phase change and the regulation of trichome distribution in Arabidopsis thaliana. Development, 1997, 124: 645-654
41 Shepherd RW, Bass WT, Houtz RL, Wagner GJ. Phylloplanins of tobacco are defensive proteins deployed on aerial surfaces by short glandular trichomes. Plant Cell, 2005, 17: 1851-1861

42 Wagner GJ. Secreting glandular trichomes: more than just hairs. Plant Physiol, 1991, 96: 675-679

43 Salinas M, Xing S, Hohmann S, Berndtgen R, Huijser P. Genomic organization, phylogenetic comparison and differential expression of the SBP-box family of transcription factors in tomato. Planta, 2012, 235: 1171-1184

44 Franco-Zorrilla JM, Valli A, Todesco M, Mateos I, Puga MI, Rubio-Somoza I, Leyva A, Weigel D, Garcia JA, Paz-Ares J. Target mimicry provides a new mechanism for regulation of microRNA activity. Nat Genet, 2007, 39: 1033-1037

45 Jiao Y, Wang Y, Xue D, Wang J, Yan M, Liu G, Dong G, Zeng D, Lu Z, Zhu X, Qian Q, Li J. Regulation of OsSPL14 by OsmiR156 defines ideal plant architecture in rice. Nat Genet, 2010, 42: 541-544

46 Miura K, Ikeda M, Matsubara A, Song XJ, Ito M, Asano K, Matsuoka M, Kitano H, Ashikari M. OsSPL14 promotes panicle branching and higher grain productivity in rice. Nat Genet, 2010, 42: 545-549

47 Schwab R, Palatnik JF, Riester M, Schommer C, Schmid M, Weigel D. Specific effects of microRNAs on the plant transcriptome. Dev Cell, 2005, 8: 517-527

48 Ferreira e Silva GF, Silva EM, Azevedo Mda S, Guivin MA, Ramiro DA, Figueiredo CR, Carrer H, Peres LE, Nogueira FT. microRNA156-targeted SPL/SBP box transcription factors regulate tomato ovary and fruit development. Plant J, 2014, 78: 604-618

49 Wang JW. Regulation of flowering time by the miR156-mediated age pathway. J Exp Bot, 2014, 65: 4723-4730

Open Access This article is distributed under the terms of the Creative Commons Attribution License which permits any use, distribution, and reproduction in any medium, provided the original author(s) and source are credited.

\section{Supporting Information}

Figure S1 Phylogenetic analysis of NtSPLs and SlySBPs.

Figure S2 miR156 binding site in NtSPLs.

The supporting information is available online at life.scichina.com and link.springer.com. The supporting materials are published as submitted, without typesetting or editing. The responsibility for scientific accuracy and content remains entirely with the authors. 